\title{
Comparison of Clinical Severity of Congenital Muscular Torticollis Based on the Method of Child Birth
}

\author{
Seung Jae Lee, M.D., Jae Deok Han, M.D., Han Byul Lee, M.D., Jee Hyun Hwang, M.D., \\ Se Yon Kim, M.D., Myong Chul Park, M.D. ${ }^{1}$, Shin-Young Yim, M.D.
}

Departments of Physical Medicine and Rehabilitation, ${ }^{1}$ Plastic and Reconstructive Surgery, Ajou University School of Medicine, Suwon 442-749, Korea

\begin{abstract}
Objective To compare the clinical severity of congenital muscular torticollis (CMT) based on the method of child birth.

Method Children diagnosed with CMT and who were $<6$-years-of-age at the time of their first visit at the Center for Torticollis, Ajou Medical Center, were included in this study. The medical records were retrospectively reviewed with reference to the method of child birth and the clinical severity of CMT. The clinical severity of CMT was determined either by whether stretching exercises were needed for the children $<6$-month-of-age or whether surgical release was required for the children $\geq 6$-months-of-age at the time of the first visit.

Results One hundred seventy eight subjects with CMT were enrolled. There was no significant difference in the rate of surgical release according to the method of child birth. For 132 patients $<6$-month-of-age there was also no significant difference in the rate of stretching exercises.

Conclusion There was no significant difference in the clinical severity of CMT based on the method of child birth. This finding suggests that prenatal factors alone could be a cause of CMT and that the clinical severity of CMT in children delivered by Cesarean section is not different when compared with the severity of CMT in children born through vaginal delivery.
\end{abstract}

Key Words Congenital torticollis, Vaginal delivery, Cesarean section

Received January 30, 2011; Accepted July 18, 2011

Corresponding author: Shin-Young Yim

Department of Physical Medicine and Rehabilitation, Ajou University School of Medicine, San 5th, Wonchon-dong, Yeongtong-gu, Suwon 442749 , Korea

Tel: +82-31-219-5284, Fax: +82-31-219-5209, E-mail: syyim@ajou.ac.kr (c) This is an open-access article distributed under the terms of the Creative Commons Attribution Non-Commercial License (http://creativecommons.org/ licenses/by-nc/3.0) which permits unrestricted noncommercial use, distribution, and reproduction in any medium, provided the original work is properly cited. Copyright (๑) 2011 by Korean Academy of Rehabilitation Medicine

\section{INTRODUCTION}

Congenital muscular torticollis (CMT) is a common musculoskeletal anomaly in infants, with the reported incidence being $0.084-3.92 \% .{ }^{1,2}$ The characteristic major clinical feature is the thickening and shortening of the sternocleidomastoid (SCM) muscle, which leads to head tilt and limited head rotation. Some infants with CMT have a cough reflex while rotating the head to the affected side. ${ }^{3}$ Skull asymmetry or plagiocephaly may occur in the presence of prolonged uncorrected CMT. Furthermore, 
facial asymmetry and scoliosis may also develop and become aggravated as the patient grows. ${ }^{4,5}$

The traditional birth trauma theory proposes that the SCM muscle is torn at birth. However, the true etiology remains uncertain. Other suggestions are intrauterine malposition, ischemic hypothesis, the intrauterine or perinatal compartment syndrome theory and the hereditary hypothesis. ${ }^{6-9}$ Considering these varied theories, CMT is best viewed as a group of clinical presentations that are characterized by neck deformity that primarily involves shortening of the SCM muscle caused by various prenatal or perinatal etiologies. Concurrent hip dysplasia in $5-10 \%$ of patients with $\mathrm{CMT}^{10,11}$ supports intrauterine malposition as a possible etiology. However, research on the prenatal etiology of CMT is limited. In addition, CMT is seen in patients who are born by cesarean section; these patients present neck magnetic resonance imaging (MRI) findings of CMT that are commonly seen in patients who are born by normal vaginal delivery (Fig. 1).
We hypothesized that, if the clinical severity of CMT does not differ based on the method of delivery, then CMT can develop by prenatal factors. In this study, the clinical severity of CMT was studied based on the method of delivery with the aim of determining the role of prenatal factors on the development of CMT.

\section{MATERIALS AND METHODS}

\section{Subjects}

One hundred seventy eight patients $<6$-years-of-age and who were followed for more than 6 months in our Center for Torticollis following a diagnosis of CMT from January, 2009 to December, 2009 were included in this study. CMT was diagnosed when the difference of the thickness of the SCM muscle on both sides exceeded $2 \mathrm{~mm}$ on ultrasonography or MRI of the neck. ${ }^{3}$ We excluded the patients with abnormal postures of the head and neck as related to ocular causes, neurodevelopmental disorders such as cerebral palsy, intellectual disability and other
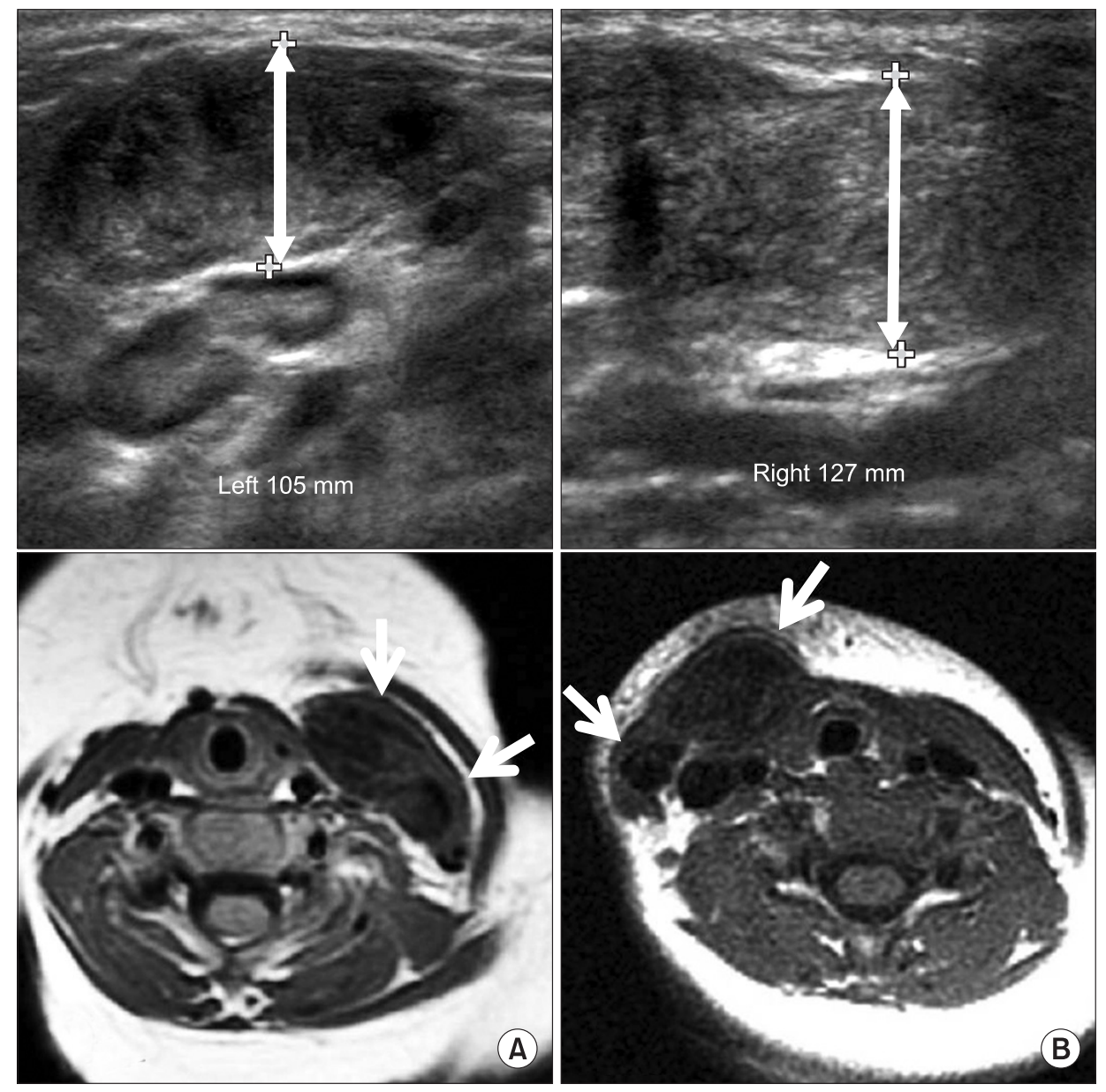

Fig. 1. Neck ultrasonography and MRI of the subjects who were born via either vaginal delivery (A) or cesarean section (B). (A) The ultrasonography and axial T1-weighted MRI of an 8-monthold boy with left CMT shows enlargement of the left SCM and low signal intensities (arrows). (B) The ultrasonography and axial T1weighted MRI of a 9-month-old girl with right CMT shows enlargement of the right SCM and low signal intensities (arrows). 


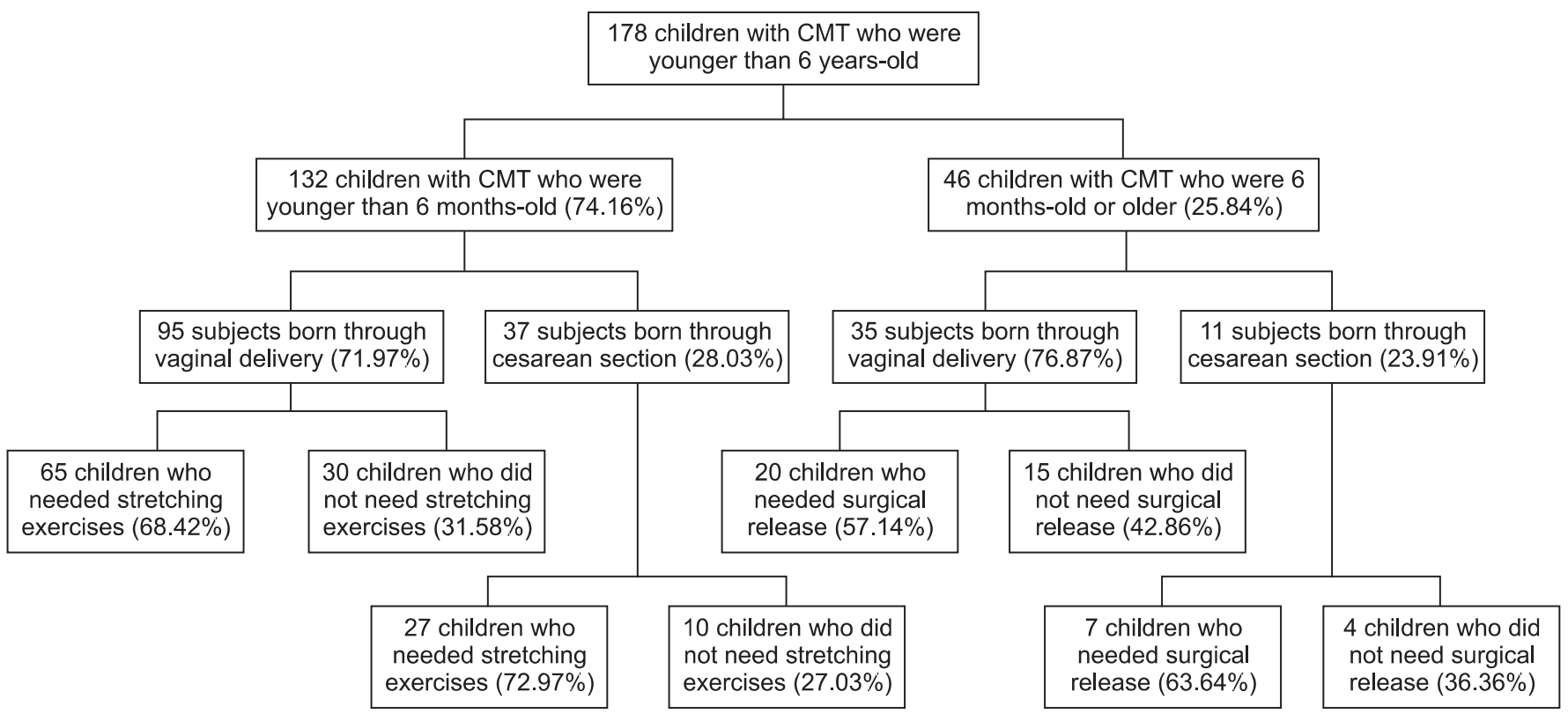

Fig. 2. The clinical pathways for the treatment of children with CMT.

conditions such as inflammation or infections of the neck or, structural abnormalities of the cervical spine.

We retrospectively reviewed the medical records of the 178 study participants and classified the patients to a vaginal delivery group and a cesarean section group. We collected the clinical information for all the study participants, including the age at the first visit, the gestational age, the birth weight, the ultrasonographic findings and MRI findings (Fig. 2).

To evaluate the clinical severity of CMT, the need for stretching exercise was used as an index for patients $<6$-months-of-age and the need for surgery was used as an index for patients $\geq 6$-months-of-age.

\section{Therapeutic protocol for CMT}

The therapeutic protocol followed two age-related clinical pathways for CMT that have been instituted at the Center for Torticollis, Ajou Medical Center (Fig. 3). These are described in detail below.

Therapeutic protocol for patients $<6$-months-ofage: One protocol was for patients $<6$-months-of-age at their first visit. For patients without resistance when performing range-of-motion (ROM) exercise, outpatient department follow-up was done without manual stretching exercise. Manual stretching was performed for patients with a shortened SCM muscle and resistance during ROM exercise. Twenty minutes manual stretching of the tight SCM was performed three-to-five times a week by an experienced physiotherapist who was properly trained and who had more than 10 years of experience. Each session consisted of applied ultrasound to the enlarged and shortened SCM, followed by massage and manual stretches. Caregivers were taught stretching exercises and were encouraged to provide more than one session of 10-minute manual stretching exercise a day at home. The treatment was considered successful and was terminated when the full ROM was restored without shortening of the SCM muscle and there was no resistance during rotation and lateral flexion. MRI was done for patients who still had resistance to motion of the neck, a thickened SCM muscle or progressive skull or facial asymmetry after 6 weeks of stretching exercise. If definite low signal intensities on the shortened SCM muscle were noted on the T1 weighted image (Fig. 1) or manual stretching could not be done due to the patient's poor cooperation despite moderate resistance on ROM, surgical treatment was done after 6-months-of-age. An incision was placed transversely at $1.5 \mathrm{~cm}$ above the clavicle on the affected side. The SCM muscle was then cleared from the underlying fascia, and the tight band was completely divided by cautery, and the abnormal fibrous band was excised at the clavicular and/or sternal head. 


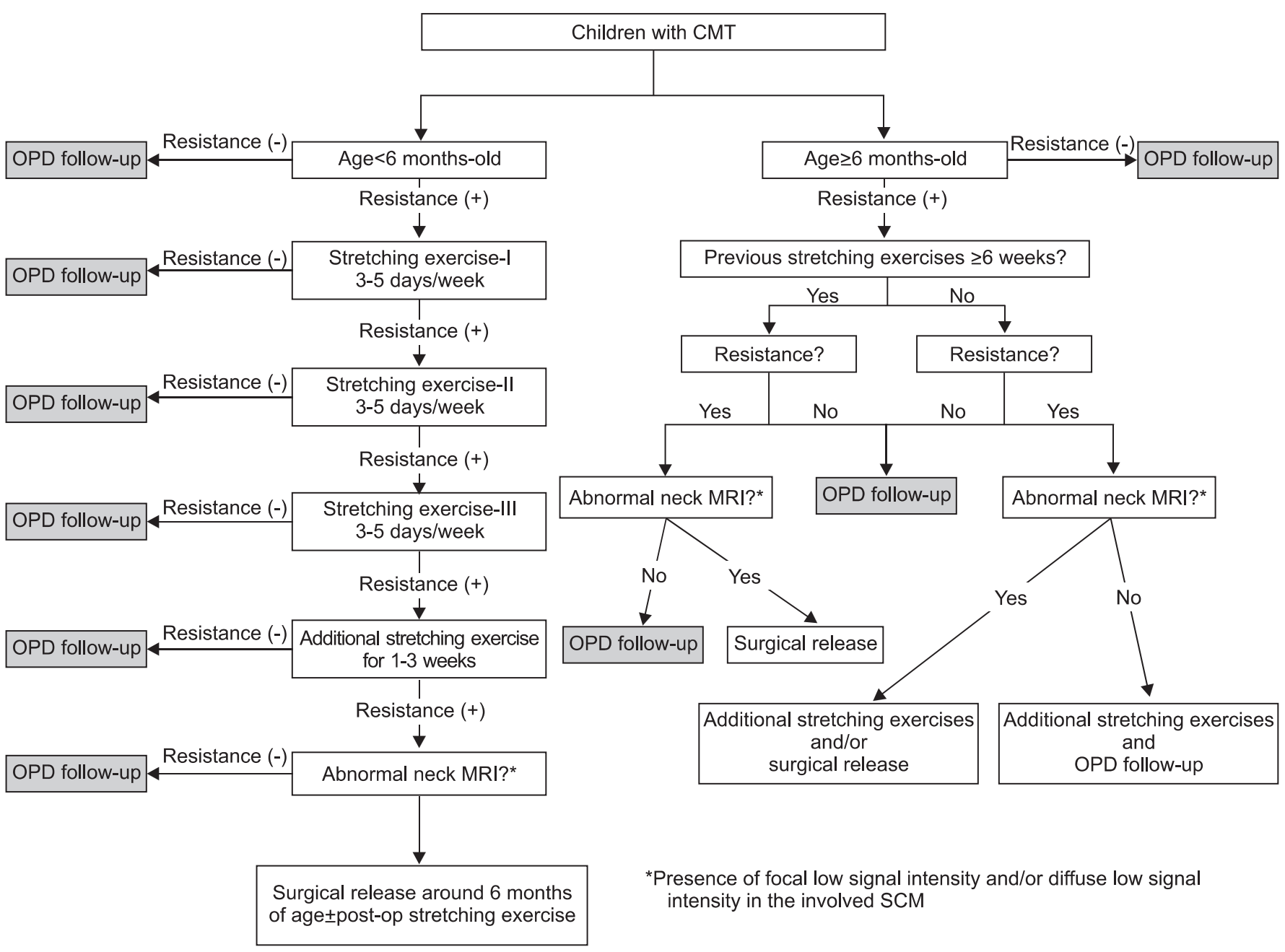

Fig. 3. The enrollment algorithm for the subjects from January 2009 to December 2009.

Therapeutic protocol for patients $\geq 6$-months-ofage: MRI was done for patients who still had resistance to motion of the neck, a thickened SCM muscle or progressive skull or facial asymmetry even after more than 6 weeks of physical therapy in other hospitals. If definite low signal intensities on the shortened SCM muscle were noted on the T1 weighted image, then surgical treatment was done. In addition, patients who had never undergone physical therapy received surgery if there was resistance on motion of neck with definite low signal intensities seen on the T1 weighted image (Fig. 1) and difficulties on stretching exercise. For those patients who had never undergone physical therapy at other hospitals, manual stretching was performed on patients with resistance on motion, but who were without definite low signal intensity.

\section{Statistical analysis}

We tested the differences among the groups with the $t$ test using SPSS 11.5 for windows for such variables as the age at the first visit, the gestational age and the birth weight. We used Fisher's Exact test to compare the necessity of surgical release and stretching exercise of each group. A p-value $\leq 0.05$ was considered significant.

\section{RESULTS}

\section{Comparison between the two groups}

Of the 178 CMT patients (98 boys and 80 girls), there were 132 patients $(74.16 \%)$ in the vaginal delivery group was and 46 patients $(25.84 \%)$ in the cesarean section group. There was no significant difference in the age at the first visit, gestational age and birth weight between the two groups (Table $1, \mathrm{p}>0.05$ ). The data showed that 
Table 1. Characteristics of the Children with CMT and Who Were younger than 6 Years-old Based on the Method of Child Birth*

\begin{tabular}{lccc}
\hline \multicolumn{1}{c}{ Characteristics } & $\begin{array}{c}\text { Vaginal } \\
\text { delivery }\end{array}$ & $\begin{array}{c}\text { Cesarean } \\
\text { section }\end{array}$ & p-value \\
\hline Number of subjects (\%) & $132(74.16)$ & $46(25.84)$ & 0.000 \\
\hline Age at the first visit (months-old) $^{\dagger}$ & $5.84 \pm 9.15$ & $5.45 \pm 9.59$ & 0.824 \\
\hline $\begin{array}{l}\text { Gestational age (weeks) } \\
\text { Birth weight (gram) }^{\dagger}\end{array}$ & $38.97 \pm 2.90$ & $38.79 \pm 1.12$ & 0.335 \\
\hline $\begin{array}{l}\text { Percentage of subjects who needed stretching exercises for CMT } \\
\text { (number of subjects) }\end{array}$ & $3,302 \pm 385$ & $3,173 \pm 439$ & 0.292 \\
\hline $\begin{array}{l}\text { Percentage of subjects who needed surgical release for CMT } \\
\text { (number of subjects) }\end{array}$ & $60.77(79)$ & $64.58(31)$ & 0.729 \\
\hline
\end{tabular}

${ }^{*}<6$-years-of-age, $\mathrm{n}=178 .{ }^{\dagger}$ mean \pm standard deviation

Table 2. Characteristics of the Children with CMT and Who Were younger than 6 Months-old Based on the Method of Child Birth*

\begin{tabular}{lccc}
\hline \multicolumn{1}{c}{ Characteristics } & $\begin{array}{c}\text { Vaginal } \\
\text { delivery }\end{array}$ & $\begin{array}{c}\text { Cesarean } \\
\text { section }\end{array}$ & p-value \\
\hline Number of subjects (\%) & $95(71.97)$ & $37(28.03)$ & 0.000 \\
\hline Age at the first visit (months-old) ${ }^{\dagger}$ & $1.39 \pm 1.01$ & $1.40 \pm 1.07$ & 0.785 \\
\hline Gestational age (weeks) $^{\dagger}$ & $38.93 \pm 3.27$ & $38.68 \pm 1.08$ & 0.348 \\
\hline $\begin{array}{l}\text { Birth weight (gram) } \\
\text { Percentage of subjects who needed stretching exercises for CMT } \\
\text { (number of subjects) }\end{array}$ & $3,300 \pm 395$ & $3,204 \pm 451$ & 0.314 \\
\hline
\end{tabular}

*<6-months-of-age, $\mathrm{n}=132 .{ }^{\dagger}$ mean \pm standard deviation

Table 3. Characteristics of the Children with CMT and Who Were 6 Months-old or Older Based on the Method of Child Birth*

\begin{tabular}{lccc}
\hline \multicolumn{1}{c}{ Characteristics } & $\begin{array}{c}\text { Vaginal } \\
\text { delivery }\end{array}$ & $\begin{array}{c}\text { Cesarean } \\
\text { section }\end{array}$ & p-value \\
\hline Number of subjects (\%) & $35(76.87)$ & $11(23.91)$ & 0.000 \\
\hline Age at the first visit (months-old) $^{\dagger}$ & $17.91 \pm 10.46$ & $19.09 \pm 12.74$ & 0.727 \\
Gestational age (weeks) $^{\dagger}$ & $39.09 \pm 1.54$ & $39.20 \pm 1.23$ & 0.587 \\
Birth weight (gram) $^{\dagger}$ & $3,305 \pm 364$ & $3,066 \pm 400$ & 0.912 \\
$\begin{array}{l}\text { Percentage of subjects who needed surgical release for CMT } \\
\text { (number of subjects) }\end{array}$ & $57.1(20)$ & $63.6(7)$ & 1.000 \\
\hline
\end{tabular}

${ }^{*} 6$ months-old $\leq$ age $<6$ years-old, $\mathrm{n}=46 .{ }^{\dagger}$ mean \pm standard deviation

$60.77 \%$ of the vaginal delivery group and $64.58 \%$ of the cesarean section group needed manual stretching exercise. Surgical release was needed for $20.76 \%$ of the vaginal delivery group and for $20.83 \%$ of the cesarean section group; the difference was not significant (Table 1 , $\mathrm{p}>0.05$ ).

\section{Comparison of CMT clinical severity for patients} <6-months-of-age

Of the 132 patients <6-months-of-age, $95(71.97 \%)$ were in the vaginal delivery group and $37(28.03 \%)$ were in the cesarean section group. No significant differences were noted for the variables, including the age at the first visit, gestational age and birth weight (Table $2, \mathrm{p}>0.05$ ). 
The number of patients who needed manual stretching exercise was $65(68.42 \%)$ in the vaginal delivery group and $27(72.97 \%)$ in the cesarean section group, respectively, and there was no significant difference (Table $2, \mathrm{p}>0.05$ ).

\section{Comparison of CMT clinical severity for the patients $\geq 6$-months-of-age}

Among the 46 patients $\geq 6$-months-of-age but $<6$-yearsof-age at their first visit, $35(76.09 \%)$ were in the vaginal delivery group and $11(21.91 \%)$ were in the cesarean section group. No significant differences were noted in the baseline characteristics between these two groups (Table 3, p $>0.05$ ). The number of patients who needed surgical release was $20(57.1 \%)$ in the vaginal delivery group and 7 (63.6\%) in the cesarean section group, and there was no significant difference (Table $3, p>0.05$ ).

\section{DISCUSSION}

Presently, there was no difference in the clinical severity of CMT according to the method of delivery. The lack of a significant difference in the frequency of manual stretching exercise or surgical release among the vaginal delivery group and the cesarean section group suggests that prenatal factors alone can cause CMT, because the cesarean section patients have little risk of birth trauma during the perinatal period.

Conventionally, the birth trauma theory has been widely suggested. This theory proposes that the SCM muscle is torn at birth with the formation of a hematoma and there is fibrous contracture. However, an experimentally-torn SCM muscle did not cause the muscle shortening or a mass, which is commonly seen in CMT. ${ }^{8}$ A study reported six cases of a familial history of CMT, with three of the cases being siblings. ${ }^{9}$ Five cases of CMT were reported in one Turkish family, including siblings, in $1997,{ }^{6}$ with a report describing CMT cases in Korean siblings. ${ }^{7}$ These reports suggested that the genetic etiology can play role in the development of CMT. ${ }^{6,7}$ These reports suggest that the genetic etiology can play role in the development of CMT. It has been proposed that CMT could be the basis of intrauterine or perinatal compartment syndrome, based on injecting radio-opaque material into the SCM. ${ }^{12}$ Another possible hypothesis is that CMT is caused by intrauterine malposition and insufficient space. ${ }^{8,13}$ This theory is supported by the fact that $5-10 \%$ of CMT children have coexistent hip dysplasia and $6.5 \%$ have other musculoskeletal anomalies such as equinovarus. ${ }^{10}$ The ischemic hypothesis, which postulates that venous occlusion by intrauterine malposition produces focal hypoxic-ischemia in the SCM muscle, has not yet been clearly proven. The selective occurrence of CMT in SCM muscle among many other neck muscles has not yet been explained. ${ }^{12,14}$ Although diffuse proliferation of fibroblasts, fibrosis and atrophy of myocytes are histologically apparent in CMT, no histological differences associated with pathogenesis have been described. Therefore, studies on the etiology of CMT from various points of views are needed.

Presently, no difference of the clinical severity depending on the method of delivery was evident. This suggests that prenatal factors by themselves can cause CMT without birth trauma. A large prospective cohort study with prenatal ultrasonographic evaluation of fetal positioning and long term follow-up is needed to obtain more details on the causal role of prenatal factors in CMT. Some children presently displayed low signal intensities in the shortened SCM muscle on T1 weighted images. Further study is also needed for determining the correlation between the severity of CMT on imaging and the prognosis.

This study has several limitations. First, it was retrospective. Second, although patients were delivered by cesarean section, there were no records on the nature of the cesarean surgery (emergency or elective), the presence of dystocia and if excessive stretching of the neck during the cesarean section occurred. So, the possibility of perinatal trauma on the SCM muscle cannot be completely excluded, even for the cesarean section group. However, the relatively large sample size and the consistency of results among different age group enhance the importance of our study.

\section{CONCLUSION}

CMT can develop by prenatal factors alone and the importance of prenatal factors should be emphasized. A large prospective study is needed for more exact assessment of the pertinent prenatal factors of CMT.

\section{REFERENCES}

1. Chen MM, Chang HC, Hsieh CF, Yen MF, Chen TH. Predictive model for congenital muscular torticollis: 
analysis of 1021 infants with sonography. Arch Phys Med Rehabil 2005; 86: 2199-2203

2. Hsu TC, Wang CL, Wong MK, Hsu KH, Tang FT, Chen HT. Correlation of clinical and ultrasonographic features in congenital muscular torticollis. Arch Phys Med Rehabil 1999; 80: 637-641

3. Yim SY, Lee IY, Cho KH, Kim JK, Lee IJ, Park MC. The laryngeal cough reflex in congenital muscular torticollis: is it a new finding? Am J Phys Med Rehabil 2010; 89: 147-152

4. Binder H, Eng GD, Gaiser JF, Koch B. Congenital muscular torticollis: results of conservative management with long-term follow-up in 85 cases. Arch Phys Med Rehabil 1987; 68: 222-225

5. Canale ST, Griffin DW, Hubbard CN. Congenital muscular torticollis. A long-term follow-up. J Bone Joint Surg Am 1982; 64: 810-816

6. Cho KH, Kim JY, Lee IY, Yim SY. Congenital muscular torticollis in siblings: a case report and literature review. J Korean Acad Rehab Med 2009; 33: 731-734

7. Engin C, Yavuz SS, Sahin FI. Congenital muscular torticollis: is heredity a possible factor in a family with five torticollis patients in three generations? Plast Reconstr Surg 1997; 99: 1147-1150
8. Lidge RT, Bechtol RC, Lambert CN. Congenital muscular torticollis; etiology and pathology. J Bone Joint Surg Am 1957; 39-A: 1165-1182

9. Suzuki S, Yamamuro T, Fujita A. The aetiological relationship between congenital torticollis and obstetrical paralysis. Int Orthop 1984; 8: 175-181

10. Cheng JC, Tang SP, Chen TM, Wong MW, Wong EM. The clinical presentation and outcome of treatment of congenital muscular torticollis in infants--study of 1,086 cases. J Pediatr Surg 2000; 35: 1091-1096

11. Hummer CD, MacEwen GD. The coexistence of torticollis and congenital dysplasia of the hip. J Bone Joint Surg Am 1972; 54: 1255-1256

12. Davids JR, Wenger DR, Mubarak SJ. Congenital muscular torticollis: sequela of intrauterine or perinatal compartment syndrome. J Pediatr Orthop 1993; 13: 141-147

13. Do TT. Congenital muscular torticollis: current concepts and review of treatment. Curr Opin Pediatr 2006; 18: 26-29

14. Yim SY, Lee IY, Park MC, Kim JH. Differential diagnosis and management of abnormal posture of the head and neck. J Korean Med Assoc 2009; 52: 705-718 